\title{
The MeerKAT Radio Telescope
}

\author{
Justin L. Jonas ${ }^{* a b}$ and the MeerKAT Team ${ }^{b}$ \\ ${ }^{a}$ Rhodes University \\ ${ }^{b}$ SKA South Africa \\ E-mail: J.Jonas@ru.ac.za
}

This paper is a high-level description of the development, implementation and initial testing of the MeerKAT radio telescope and its subsystems. The rationale for the design and technology choices is presented in the context of the requirements of the MeerKAT Large-scale Survey Projects. A technical overview is provided for each of the major telescope elements, and key specifications for these components and the overall system are introduced. The results of selected receptor qualification tests are presented to illustrate that the MeerKAT receptor exceeds the original design goals by a significant margin.

MeerKAT Science: On the Pathway to the SKA,

25-27 May, 2016,

Stellenbosch, South Africa

${ }^{*}$ Speaker. 


\section{Introduction}

The MeerKAT radio telescope is a precursor for the Square Kilometre Array (SKA) midfrequency telescope, located in the arid Karoo region of the Northern Cape Province in South Africa. It will be the most sensitive decimetre-wavelength radio interferometer array in the world before the advent of SKA1-mid. The telescope and its associated infrastructure is funded by the government of South Africa through the National Research Foundation (NRF), an agency of the Department of Science and Technology (DST). Construction and commissioning of the telescope has been the responsibility of the SKA South Africa Project Office, which is a business unit of the NRF. Telescope operations will be the responsibility of the newly declared South African Radio Astronomy Observatory (SARAO), a National Facility administered by the NRF. MeerKAT will be incorporated into the SKA1-mid array towards the end of the construction phase of SKA1-mid.

The MeerKAT project resulted from a DST strategy for South Africa to develop appropriate technical and scientific capabilities in the academic and industrial sectors in order to be able to participate fully in the international SKA project. A range of technology prototyping activities were undertaken prior to embarking on the final design of MeerKAT. These involved the investigation of novel technologies for use in the SKA and MeerKAT, including phased-array feeds (PAFs), ambient temperature receiver clusters, single-piece composite reflectors, RF-over-fibre analog signal transport and Stirling-cycle cryogenic coolers. Two prototype telescopes were designed, constructed and commissioned as part of the prototyping programme: the single $15 \mathrm{~m}$ XDM dish at HartRAO and the seven element KAT-7 interferometer array in the Karoo. Both of these prototype telescopes have been used for productive science programmes.

The full MeerKAT array with 64 antennas is scheduled to start early science operations mid 2018. The full deployment will be preceded by a series of array releases as antennas are added to the array, and the functionality of the correlator/beamformer, science processor and control software ramps up. The schedule for this staged implementation is dynamic - the current state of actual and planned roll-out is to be found at:

http://public.ska.ac.za/meerkat/meerkat-schedule.

At the time of writing the 16-element Array Release 1 (AR1) has successfully demonstrated the ability to: (1) perform deep, wide-band, wide-field (mosaic) continuum imaging of extragalactic fields and extended sources in the Galactic plane, (2) perform narrow-band imaging of Galactic $\mathrm{OH}$ maser sources, (3) produce neutral hydrogen (HI) data cubes for nearby galaxies and galaxy clusters, (4) monitor pulsar times of arrival, and (5) detect a pulsar.

\section{The MeerKAT Large (Legacy) Survey Projects}

When the DST made the decision to fund the design and construction of a substantial SKA precursor instrument in the Karoo, a directive was issued that the instrument must have transformational science goals related to those of the SKA, and that the technologies used to implement the instrument should be applicable to the eventual SKA. A preliminary MeerKAT science programme was formulated in consultation with a broad range of scientists associated with the development of the SKA science case, and this programme was distilled into a set of high level technical require- 
ments for the MeerKAT telescope. The preliminary MeerKAT science case is outlined in [1], and an early MeerKAT reference design concept is described in [2].

In 2009 a call was made to the international science community to self-organize into project teams and propose large-scale survey projects (LSPs) that might be carried out on the MeerKAT telescope as defined in [1]. A large-scale survey was defined as requiring in excess of 1000 hours of observing time, and these surveys would nominally occupy $75 \%$ of the first 5 years of MeerKAT full-scale operations. This solicitation resulted in 20 survey proposals that were adjudicated by a Time Allocation Committee (TAC). Eleven of the proposals were approved, with two of them recommended for merger because of the similarity in science objectives and survey definition. The resulting ten LSPs approved for observing time are described very briefly below.

\subsection{LADUMA}

An ultra-deep atomic hydrogen (HI) emission survey out to a redshift of 1.4 to investigate the cosmic evolution of $\mathrm{HI}$ in galaxies over the last $9 \mathrm{Gyr}$. The observations will be towards one single pointing within the Chandra Deep Field South, using both the UHF and L-band receivers to cover the redshift range.

\subsection{MIGHTEE}

An extra-galactic continuum survey to investigate the evolution of AGN, star-forming galaxies and galaxy clusters from the epoch of reionization through to the present day. These observations of four fields, combined with extensive ancillary multi-wavelength data, will also probe the evolution of cosmic magnetism.

\subsection{MHONGOOSE}

An HI survey to map the distribution and dynamics of neutral hydrogen in a sample of 30 galaxies located within $30 \mathrm{Mpc}$ and spanning five orders of magnitude in HI mass. MeerKAT will uniquely have the capability to detect and make detailed maps of the faint gas falling into galaxies from their surroundings.

\subsection{Fornax}

A deep HI emission line survey of the Fornax cluster to study the dynamics of neutral gas in the cluster and its connection to the cosmic web. These observations of the largest nearby $(20 \mathrm{Mpc})$ cluster in the southern hemisphere should yield $\sim 100 \mathrm{HI}$ detections, probing gas dynamics at a spatial resolution of $\sim 1 \mathrm{kpc}$, and the cosmic web at a resolution of $\sim 10 \mathrm{kpc}$.

\subsection{MALS}

An extremely sensitive survey of $\mathrm{HI}$ and $\mathrm{OH}$ absorbers that will map the evolution of cold atomic and molecular gas in galaxies at $0 \leq z \leq 2$. This redshift range probes the evolution in star-formation rate density from near its peak at $z \approx 2$ to the low levels observed in the current universe. 


\subsection{ThunderKAT}

A program to make image-plane detections of multiple populations of Galactic and extraGalactic synchrotron transient radio sources associated with high energy accretion and explosive events. This monitoring, target-of-opportunity, and commensal program will often be done in concert with observations at other wavelengths.

\subsection{MeerGAL}

The deepest and highest resolution $14 \mathrm{GHz}$ survey of the southern Galactic plane, aiming to probe Galactic structure and dynamics, including the study of hyper-compact HII regions and anomalous microwave emission from small spinning dust grains.

\subsection{MESMER}

A survey targeting redshifted emission originating from the $J(1-0)$ transition of $12 \mathrm{CO}$ associated with molecular gas in high-redshift $(z \approx 7)$ galaxies, to investigate the role of molecular hydrogen in the early universe.

\subsection{Pulsar Timing (MeerTime)}

Long-term timing of various classes of pulsars for a range of scientific goals, including the detection of the gravitational wave background, tests of General Relativity and alternative theories of gravity, probing binary evolution, and constraining the structure of nuclear matter.

\subsection{TRAPUM}

Targeted and blind surveys for the detection of fast radio transients and pulsars. The expected discovery of large numbers of Fast Radio Bursts and of pulsars in targets such as unidentified Fermi sources, supernova remnants, and globular clusters, will contribute to the understanding of physics in extreme environments and of Galactic dynamics.

\subsection{Proposals approved, but not allocated observing time}

The MeerQuittens polarization rotation measure survey to probe the magnetic fields of galaxies, galaxy groups and galaxy clusters was not allocated observing time, but the TAC suggested instead the commensal use of data obtained by other surveys. The proposal to conduct VLBI observations with MeerKAT was not considered to be in the category of large surveys, and the TAC recommended that VLBI observations be scheduled using the common practice at other major observatories.

\section{Concept and Baseline Design}

The science requirements derived from the ten MeerKAT large survey proposals were used to refine the initial telescope specifications provided in the call for proposals [1]. The specifications and reference design concept of the MeerKAT telescope have evolved further since this early 
conceptualization. The evolution of the reference design was the outcome of a detailed and extensive exploration of concepts and technologies intended to optimize the scientific impact of the instrument, moderated by the realities of a fixed budget and schedule.

The antenna diameter, optical configuration, cryogenic cooling technology and receiver technology were determined though a constrained joint electromagnetic and mechanical optimization process. The goal was to maximize point-source sensitivity under the constraints of capital and operational costs. A secondary goal of the optimization was to maximize imaging dynamic range. Given the difficulty of deriving definitive system specifications from a dynamic range requirement, a pragmatic strategy was adopted where the Jones matrices associated with all components in the signal path were specified to be stable and smooth over time, frequency and direction. The expectation is that this will assist tractable calibration algorithms to achieve the required dynamic range. This strategy placed tight constraints on the antenna primary beam characteristics (near and far sidelobe levels, circular symmetry, cross-polarization at boresight and within the primary beam) and the RF signal path (passband flatness and stability). Operational issues, such as access to the receivers and the physical volume available for receivers, were also considered in the optimization.

Formal system design reviews, including a Concept Design Review (CoDR) and a Preliminary Design Review (PDR), were convened to obtain guidance and reassurance from expert panels. The panels were chosen to harness the experience of individuals who had been involved in similar large projects, such as ALMA, ATCA, WRST, eMERLIN and the JVLA. Key charges assigned to these panels were to assess performance risks, technology maturity and costing fidelity of the designs presented.

Key changes to the early MeerKAT concept described in [1] include:

- Changing the antenna optical configuration from a $12 \mathrm{~m}$ symmetric centre-fed dish to a (nominal) $13.5 \mathrm{~m}$ offset Gregorian dish. The motivation for this change is given in section 4.2.

- Reduction of the number of antennas from 80 to 64 (thus retaining the original aggregate physical aperture with the larger dishes).

- Limiting the maximum array baseline to $\sim 8 \mathrm{~km}$. A $60 \mathrm{~km}$ "spur" was part of the original concept, but this was excluded on the basis of cost and feasibility issues.

- The use of two-stage Gifford-McMahon (G-M) cryogenic coolers in the receiver systems, as opposed to the original concept of using single-stage Stirling-cycle systems. The motivation for this decision is given in section 4.3.

- The use of sub-octave band receiver systems in place of wide-band receivers. The motivation for this decision is given in section 4.3.

The 2009 array configuration was a compromise between the requirement of a compact core for observing pulsars and extended, low-brightness sources, and the requirement of a scale-free antenna distribution for medium-resolution, high dynamic range imaging [1]. This configuration provides a flat sensitivity characteristic for angular scales between $8^{\prime \prime}$ and $80^{\prime \prime}$ at $1420 \mathrm{MHz}$ (equivalent to a simultaneous hybrid of JVLA C and D configurations), a 50\% sensitivity degradation at the highest resolution of $6^{\prime \prime}$ (equivalent to a slightly truncated JVLA B configuration), and a gradual 
degradation of sensitivity for extended structures larger than $80^{\prime \prime}$ (equivalent to the proposed JVLA E configuration). In reducing the number of array stations from 80 to 64 the same criteria were applied when optimizing the receptor positions, and the angular scale sensitivity characteristic of the reduced array is essentially identical to the original. The aggregate effective aperture of the 64 element array exceeds that of the original 80 element array because of the reduced of aperture blockage of the offset Gregorian antennas. The physical positions of the 64 MeerKAT antennas are listed in table 1 (more accurate positions will be published once all baseline calibrations being conducted during commissioning have been completed). The MeerKAT antennas will be integrated into SKA1-mid towards the end of the construction phase of the larger array. The SKA1-mid array core is centred on the MeerKAT array core, and the MeerKAT antennas will contribute a significant fraction of the SKA1-mid aperture within the core.

Because of programmatic and financial constraints, the current MeerKAT implementation plan only allows for two receivers that cover the frequency range $580 \mathrm{MHz}$ to $1670 \mathrm{MHz}$ in two overlapping bands: (1) UHF-band: $580 \mathrm{MHz}-1015 \mathrm{MHz}$, and (2) L-band: $900 \mathrm{MHz}-1670 \mathrm{MHz}$. S-band receivers are being provided by the Max Planck Institute for Radio Astronomy (MPIfR) that will cover the $1750 \mathrm{MHz}-3500 \mathrm{MHz}$ frequency band (no further details of this receiver are provided here). The implementation of the originally specified $\mathrm{X}$-band receiver, operating over the frequency band $8 \mathrm{GHz}$ to $14.5 \mathrm{GHz}$, depends on factors that are uncertain at this time. A key factor is the length of time that MeerKAT operates as an independent telescope prior to being included into SKA1-mid. Another consideration is the implementation plan for the SKA1-mid Band 5 receiver system that will operate over the (nominal) frequency range 4.6-13.8 GHz.

\section{Implementation}

In this section we provide high-level descriptions of the various elements of the MeerKAT telescope, together with key specifications and design considerations. This is a very abridged summary of an extensive engineering exercise that followed the principles of systems engineering, including design reviews by expert panels.

\subsection{Infrastructure}

MeerKAT infrastructure can be divided into three main categories:

1. Bulk infrastructure providing services to the site, including access roads, aircraft landing strip, electrical power supply from the ESKOM national utility grid, and fibre optic data connections.

2. On-site infrastructure directly supporting telescope operations, including dish foundations, power and fibre reticulation, power conditioning, and the Karoo Array Processor Building (KAPB). The KAPB houses a large shielded room providing EMI protection against RFI emissions from electrical and electronic equipment housed within it.

3. Infrastructure facilities supporting telescope construction, site maintenance and telescope maintenance These facilities include the forward support base at Klerefontein, workshops, dish assembly sheds, construction camps and the on-site road network. 


\begin{tabular}{|r|r|r|r|r|r|}
\hline Antenna & $X[\mathrm{~m}]$ & $Y[\mathrm{~m}]$ & Antenna & $X[\mathrm{~m}]$ & $Y[\mathrm{~m}]$ \\
\hline M000 & -8 & 27 & M032 & 461 & 412 \\
M001 & 1 & 63 & M033 & 581 & 1104 \\
M002 & -32 & 10 & M034 & 358 & 207 \\
M003 & -66 & 32 & M035 & 386 & 54 \\
M004 & -124 & -19 & M036 & 388 & -57 \\
M005 & -102 & -49 & M037 & 380 & -226 \\
M006 & -18 & -61 & M038 & 213 & -336 \\
M007 & -90 & -169 & M039 & 254 & -360 \\
M008 & -93 & -302 & M040 & -27 & -480 \\
M009 & 32 & -137 & M041 & -287 & -429 \\
M010 & 88 & -279 & M042 & -362 & -227 \\
M011 & 84 & -118 & M043 & -630 & 106 \\
M012 & 140 & -135 & M044 & -896 & 839 \\
M013 & 237 & -160 & M045 & -1832 & 503 \\
M014 & 281 & -52 & M046 & -1467 & 1996 \\
M015 & 211 & 16 & M047 & -578 & -284 \\
M016 & 288 & 49 & M048 & -2805 & 2935 \\
M017 & 200 & 123 & M049 & -3605 & 674 \\
M018 & 106 & -11 & M050 & -2052 & -612 \\
M019 & 171 & -51 & M051 & -850 & -537 \\
M020 & 97 & -66 & M052 & -593 & -918 \\
M021 & -296 & -93 & M053 & 9 & -1075 \\
M022 & -322 & 93 & M054 & 872 & -267 \\
M023 & -373 & 236 & M055 & 1201 & 333 \\
M024 & -351 & 386 & M056 & 1598 & 705 \\
M025 & -182 & 462 & M057 & 295 & 3511 \\
M026 & -99 & 253 & M058 & 2805 & 2935 \\
M027 & 41 & 212 & M059 & 3685 & 998 \\
M028 & -51 & 148 & M060 & 3419 & -1614 \\
M029 & -89 & 111 & M061 & -16 & -2099 \\
M030 & 171 & 350 & M062 & -1440 & -2280 \\
M031 & 247 & 330 & M063 & -3419 & -1614 \\
\hline & & & & & \\
\hline
\end{tabular}

Table 1: Locations of the 64 MeerKAT antennas relative to the fiducial array centre at $30^{\circ} 42^{\prime} 47^{\prime \prime} \mathrm{S}$, $21^{\circ} 26^{\prime} 38^{\prime \prime}$ E. $X$ values are positive toward east and $Y$ values are positive towards north. 
Although primarily providing for the MeerKAT needs, the infrastructure was designed with the requirements of SKA1-mid in mind. In some cases the currently installed infrastructure has the capacity to support SKA1-mid (e.g. the power lines). In others cases there is not the installed capacity to support SKA1-mid, but it is feasible to extend the existing capacity to meet the SKA1mid requirements with little disruption (e.g. the site data link has sufficient fibres to support SKA1mid, and the KAPB has space for more equipment racks and DRUPS).

Infrastructure elements that have direct bearing on the operation and performance of the MeerKAT telescope are described below.

\subsubsection{Dish Foundations}

Two key requirements for the dish foundations were stiffness (to achieve the required dish pointing accuracy) and grounding impedance (the specification was $1 \Omega$ to ensure lightning protection and EMI control). Each dish foundation consists of 8 cast-in-drilled-hole reinforced concrete piles on a square pattern (three per side) topped with a square reinforced concrete slab. The pile boreholes are drilled to varying depths, depending on the geotechnical conditions prevailing at the specific site. A steel ring-cage is cast into the foundation slab, providing a threaded stud interface for the dish pedestal flange. The ring-cage and reinforcing bars in the slab and piles are galvanicaly bonded to ensure robust lightning current paths. Buried radial copper conductors are also bonded to the reinforcing bars in order to achieve the $<1 \Omega$ earthing conductivity specification. The first foundation to be cast was used as the qualification article, with a pull-test jig being used for the stiffness qualification test. Grounding impedance acceptance tests were conducted for all 64 foundations.

\subsubsection{KAPB}

The KAPB provides three main facilities: electrical power conditioning, electronic equipment racks and staff work areas. A large fraction of the building volume is buried below natural ground level, providing EMI shielding for the electrical power systems and some thermal insulation. Minimizing EMI risks and maximizing EMI shielding were key issues considered during the design and construction of the building. The reinforcing bars in the cast concrete walls are all galvanically bonded and crossing points to implement a low-frequency Faraday cage, and all metallic fittings are galvanically bonded to earth in order to prevent electrostatic discharge (ESD) arcing. A large shielded room, fabricated using steel panels, provides $100 \mathrm{~dB}$ EMI shielding for the electronic and computing equipment associated with the correlator/beamformer, science processor, time and frequency reference sub-system, user-provided equipment, guest instruments and other auxiliary subsystems (e.g. the building management system). HVAC units keep the air temperature in the shielded compartment at $\sim 22 \mathrm{C}$. A smaller shielded room provides an $80 \mathrm{~dB}$ EMI shielding environment for an on-site laboratory/workshop and telescope control room.

\subsubsection{Electrical Power}

Power to the site is provided by three-phase $33 \mathrm{kV}$ overhead lines from an ESKOM national power utility substation near Carnarvon (this substation has been upgraded to neet the SKA1-mid power requirements). Special care was taken during design and construction to ensure that the overhead lines would not present an EMI risk due to arcing. All insulators are over-specified and a 
specialized procedure was employed when installing the power conductors. These lines are capable of delivering just over 5 MVA to the site. Electrical power conditioning systems within the KAPB are designed for $N+1$ redundancy, reducing the risk of down-time due to single points of failure. All electrical power on the site is conditioned and protected by three $(2+1$ redundancy) diesel rotary UPS (DRUPS) units located in the KAPB. These DRUPS units provide extremely stable and reliable power to the dishes and the central processing electronics in the KAPB, and apply dynamic power factor correction. Power to the dishes is provides via a buried $22 \mathrm{kV}$ ring-main cable. Transformer substations distributed around the array site provide $400 \mathrm{~V}$ power to between one and five dishes. Individual isolation transformers mounted on the foundation of each dish reduce the risk of equipment damage and down-time caused by lightning strikes on or near dish structures.

\subsubsection{Optical Fibre}

The data fibre cable from the site is strung on the $33 \mathrm{kV}$ power line poles and terminates in Carnarvon. Currently this link operates at $10 \mathrm{~Gb} / \mathrm{s}$ (constrained by the terminal equipment), but the fibre has a higher link capacity. An onward fibre link from the Carnarvon terminal connects to a major national data backbone that follows the railway line between Cape Town and Johannesburg, providing access to the SANReN national research network. SANReN provides connectivity to the MeerKAT data archive located at the Centre for High-Performance Computing (CHPC) in Cape Town.

All optical fibre links between the KAPB and the dish foundations are via ducts buried at a depth of $1.2 \mathrm{~m}$, providing thermal stability for the 1 PPS and sample clock distribution fibres. Waveguide below cutoff feed-through filters are used for fibre penetrations into both the shielded room at the KAPB and into the dish pedestal shielded compartment.

\subsection{Dish Structure}

The selection of a 13.5 metre projected diameter, "feed-down" offset Gregorian optical configuration for the MeerKAT dishes was an outcome of the system CoDR process. The key advantage of this configuration over the competing symmetric centre-fed parabola is the unblocked aperture that results in:

- A high aperture efficiency (ranging from 0.71 to 0.81 across the UHF to L-band frequencies), and hence good sensitivity.

- Low far sidelobes, providing some immunity to terrestrial and airborne RFI, and the attenuation of strong cosmic radio sources outside of the imaging field.

- An almost circularly-symmetric main beam and inner sidelobe pattern, hence a reduction in the calibration challenges provided by the primary beam rotating relative to the sky (i.e. slow variation of the time and direction dependent beam pattern Jones matrices).

- Elimination of ground radiation scattering off of feed legs into the feed, hence reducing the system temperature and thus increasing the sensitivity. 
- The reduction of internal specular reflections and associated passband ripples (i.e. a Jones matrix that is smooth over frequency).

- A large volume for locating multiple receiver systems and support services on the receiver index platform that does not compromise the clean optical path, hence increasing the utility of the telescope as a facility instrument.

These performance advantages were shown to justify the extra "per physical area" cost of the more complex structure relative to the the simpler centre-fed design. Furthermore, the "feed-down" configuration has these advantages over the "feed-up" configuration:

- Reduced spill-over from ground radiation, particularly if an extended skirt is fitted below the sub-reflector, hence reducing the system temperature and improving the sensitivity.

- Easy and safe access to the receiver systems when the antenna is stowed at its lowest elevation. Large cranes or cherry-pickers are not required for installation and maintenance.

A disadvantage of the feed-down configuration is that the minimum elevation limit is constrained to $15^{\circ}$. This limit is imposed by the geometry of the optical path and mechanical interference between the elevation structure and the pedestal tower.

The detailed optical configuration for the dish was derived using the joint application of fullwave electromagnetic simulations and mechanical finite element modelling. The configuration was optimized to reduce the sensitivity of key performance metrics, such as cross-polarization leakage cause by de-collimation of the reflectors and feeds [3], to time-varying stresses resulting from common load cases (e.g. wind loading and the elevation-dependence of gravity deformation). Deviation from the use of pure conic sections for the reflectors (i.e. "shaping") was not consider because of the added complexity of the main reflector moulding tools, and the associated cost.

High dynamic range, wide-field continuum imaging requires that the Jones matrix for the primary beam pattern remains stable over the characteristic time-scales of the visibility calibration cycle. This requirement was a key driver of the specifications for the MeerKAT dishes. Assuming that nearby ( $<7^{\circ}$ on the sky) reference sources are used to correct for residual pointing offsets due to wind and thermal deformations, the pointing accuracy specification is 5 " under "ideal" operating conditions and 25" under "normal" conditions. These specifications placed tight design constraints on various elements of the dish structure, including the axis encoders and elevation jack-screw.

The tender process for the dish structure design and construction was run on a "build to specification" basis, with the key specifications being the optical configuration, aperture phase efficiency, reflector efficiency, telescope pointing accuracy and repeatability, and compliance with strict EMI thresholds. The main elements of the successful contractor's design are (refer to Figure 1):

- An elevation over azimuth mount geometry employing a dual-pinion azimuth drive and a pre-loaded jack-screw elevation drive.

- A cylindrical pedestal tower with a conical top section. A shielded compartment that houses the servo drive electronics, antenna control unit, receiver control unit and data switches is an integral part of the pedestal. 


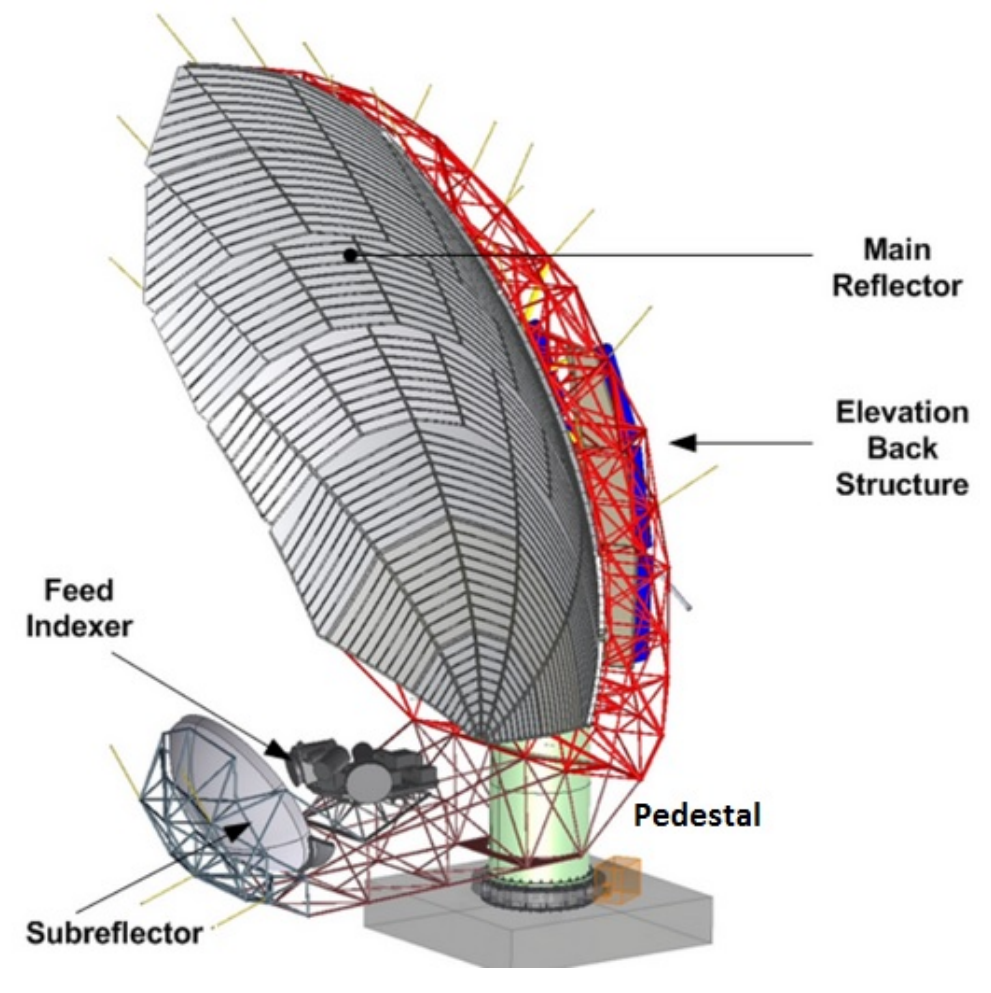

Figure 1: A schematic diagram of the MeerKAT dish structure showing the major elements of the design. The small rectangular box on the foundation cap is the isolation transformer.

- A yoke structure fabricated from steel plates that provides physical attachments for the azimuth and the elevation drives. A two-axis tilt-meter is mounted in the yoke to provide metrology data to the pointing algorithm within the antenna control unit. The closed-circuit compressor providing helium to the receiver cryogenic coolers is mounted on the exterior of the yoke.

- A primary reflector consisting of a tubular steel space-frame backup structure (BUS) supporting aluminium panels. Because the primary reflector is a pure paraboloid of revolution (i.e. unshaped), it is circularly symmetric about an apex that is near the bottom edge of the dish. The panels are arranged in seven concentric rings around this apex, with every panel in a given ring having the same figure (but not outline). This allowed for the use of seven panel moulds to fabricate all of the panels. The as-built circumference of the main reflector is piecewise linear because of the straight edges of the outer panels. This results in an effective projected diameter of 13.965 metres, hence larger than the nominal 13.5 metre specification.

- A tubular steel space-frame support boom that connects the sub-reflector and the receiver indexer to the BUS.

- A one-piece moulded composite Gregorian (elliptical) sub-reflector. The composite layup consists of kevlar, carbon fibre and aluminium foils. A conical aluminium skirt below 
the sub-reflector intersects rays from the feed horn that would otherwise point towards the ground and reflects them to the sky.

- A rotating receiver indexer platform that positions one of up to four receiver systems at the secondary focus position. In addition to the receiver packages, the receiver indexer also provides mounting pads for the digitizers, a vacuum pump that services the receiver cryostats, and optical-fibre and electrical distribution enclosures (see Figure 1).

A major design challenge was to achieve compliance with stringent EMI thresholds, driven by the very short distances between the receiver feed horns and the servo drive systems and the digital data switches located in the pedestal shielded drive compartment (SDC). These electronic components have high-speed clock signals and require penetrations through the SDC wall. High performance bulkhead filters are used for all electrical signals (e.g. 3-phase power supply, 3-phase servo motor drive, encoder signals), and waveguide below cutoff penetrations are used for fibre optic cables.

\subsection{Receivers (including Feed Horns)}

The UHF-band and L-band feed horns and receiver systems are of very similar design, with the wavelength dependent scaling of the dimensions of the waveguide and ortho-mode transducer (OMT) components being the largest difference between them. Detailed descriptions of the UHF and L-band receiver systems are to be found in [4] and [5] respectively.

The feed horns have a wide flare angle and employ concentric choke rings to control the feed beam pattern. The feed beam patterns were optimized to provide optimum SEFD, sidelobe and polarization performance across the operating frequency range in the context of the dish optical configuration . The main reason for adopting sub-octave receiver systems in preference to wideband designs was the ability to tune horn and OMT parameters to obtain optimal sensitivity and primary beam performance. Wideband systems do not allow the same degree of control. The sidelobe specifications are driven by requirements for high dynamic range continuum imaging and attenuation of RFI cosmic sources away from the field of view. For both UHF and L-band operation the second sidelobe is more than $-23 \mathrm{~dB}$ below the peak primary beam response, and all further out sidelobes are below this level. Sidelobes beyond $10^{\circ}$ from the boresight have a gain of less than $0 \mathrm{dBi}$ over $99 \%$ of the sky. The polarization specification is derived from the requirements of precision pulsar timing and high dynamic range continuum imaging. Within the $-1 \mathrm{~dB}$ contour of the primary beam the magnitude of the Jones matrix cross-polarization terms must be less than $-26 \mathrm{~dB}$ (goal $-30 \mathrm{~dB}$ ), and within the $-3 \mathrm{~dB}$ contour the magnitude of the cross-polarization terms must be less than $-20 \mathrm{~dB}$ (goal $-25 \mathrm{~dB}$ ).

The system-level optimization described earlier showed that the improvement in sensitivity resulting from the use of two-stage Gifford-McMahon (G-M) cryogenic refrigerators to cool the LNAs and lossy OMT components justified the costs associated with the complexity and power consumption. Secondary issues, such as the use of cryo-pumping to maintain cryostat vacuum, also contributed to the decision. The novel OMT and cryostat design used for the receiver package provides an integrated OMT, balun and calibration signal coupler that does not require a thermal or vacuum break in the waveguide or RF signal path, leading to reduced losses and mismatches and simplified assembly and maintenance procedures. All of these components are thermally tied to the first stage of the G-M cooler to reduce noise resulting from resistive losses in the signal 
path. The physical temperature of the crossed-dipole OMT is $\sim 90 \mathrm{~K}$ for the L-band receivers and $\sim 100 \mathrm{~K}$ for the UHF-band receivers. The design of the OMT restricts cross-polarization leakage to $\sim-30 \mathrm{~dB}$, and provides a very good inter-polarization phase match for the calibration signal.

The two front-end LNAs (one per linear polarization mode) are thermally tied to the second stage of the cryo-cooler, and are temperature stabilized at $20 \mathrm{~K}$ by heating resistors whose current is controlled by a closed-loop servo circuit. The L-band LNAs have three InP transistors and the UHFband LNAs have one InP transistor followed by two GaAs transistors. The thermal stabilization is necessary to achieve the gain stability specifications derived from the system-level requirement to achieve a high imaging dynamic range. The heating results in a small increase in the receiver noise temperature and an almost $100 \%$ increase in the second-stage heat load. This is an example of where the requirement for Jones matrix stability took precedence over raw point source sensitivity in the design process.

The calibration noise source and second stage amplifiers are mounted on a temperature-stabilized platform inside the cryostat vacuum jacket. The platform has a weak thermal path to the first stage of the cryo-cooler and is held at $\sim 300 \mathrm{~K}$ by heater resistors and a closed-loop servo circuit. The noise diode switching is controlled by the digitizer. The receiver package only provides signal gain and does not apply any RF signal conditioning.

\subsection{RF Signal Conditioning, Digitization and Digital Signal Path}

Two outcomes of the system-level optimization were that the RF signal path should be as short as possible to reduce VSWR related passband ripples, and that direct digitization without heterodyne conversion should be implemented to avoid system complexity and spurious mixer products (or "birdies"). The resulting implementation was to locate the digitizers directly behind the receiver packages on the receiver indexer platform, with a pair of short $(<1 \mathrm{~m})$ interconnecting coaxial cables transferring the RF signal from the receiver to the digitizer. Each receiver has its own dedicated digitizer, a consequence of the requirement that the digitizer sample clocks remain coherent even when the associated receiver is not rotated the secondary focus position. The location of the digitizers presented EMI and temperature challenges, but appropriate design measures have provided a successful implementation. The necessary EMI shielding is achieved by using nested shielded enclosures and fibre optic isolation of RF and digital components. Heat management is achieved through well-defined thermal paths from the heat-generating components inside the nested compartments to heatsinks on the outer casing of the digitizer. The digitizers are covered by an environmental shield that reflects direct sunlight, but allows free air-flow over the heatsinks.

The UHF and L-band digitizers are very similar in design, with the only differences being the frequency-dependent RF components (i.e. the RF conditioning circuitry and the sample clock generator). The main design drivers for the digitizers were gain stability, passband flatness and high spurious-free dynamic range (SFDR). SFDR is a measure of the strength of spurious signals appearing in the digital output of the ADCs relative to the strength of strong, narrow-band input signals (e.g. RFI signals). The digitizers have six functional units, described briefly here:

- RF conditioning unit (RFCU): This component provides analog signal conditioning and monitoring for a single polarization RF signal prior to digitization (there are two independent and isolated RFCUs per digitizer package). Amplifiers and programmable attenuators provide 
the correct signal level to the ADC, and a bandpass anti-aliasing filter defines the passband which is within Nyquist zone 2 of the ADC (A secondary advantage of sub-octave receivers is the ability to employ Nyquist down-conversion). There is no phase switch in the RF signal path because Walsh switching is not implemented on MeerKAT. Key design goals for the entire RF signal path were bandpass flatness and stability.

- Analog to digital converter (ADC): The e2v AT84AS004 10-bit device was selected for the MeerKAT digitizers because it is a single-core, non-interleaved ADC that provides excellent SFDR performance. Digitizer qualification tests have shown that the effective number of bits $(\mathrm{ENoB})$ is 7.8, which is consistent with the ADC manufacturer's specification. The ADC is electrically decoupled from all digital circuitry by digital fibre-optic links. There are two independent ADCs, one for each polarization. The ADC encodes the 1 PPS signal onto the digital voltage data stream to allow single-clock-cycle precision for time-stamping the voltage data samples.

- Packetizer (D-engine): The D-engine receives the 10-bit digital voltage data from the two ADCs via the digital fibre-optic link and frames this data into 10Gb Ethernet packets. Fibreoptic transceivers and cables transfer these packets to the receptor data switch located in the pedestal shielded compartment.

- Sample clock generator: The sample clock for the ADCs originates from the TFR subsystem (see section 4.6) in the KAPB and is transferred to each individual digitizer via a dedicated fibre. The sample clock generator converts the optical clock signal to an electrical sine-wave. A narrow-band filter constrains the phase noise to achieve the required sample clock jitter specification. The sample clock frequencies for the UHF and L-band ADCs are $1088 \mathrm{MHz}$ and $1712 \mathrm{MHz}$ respectively.

- Pulse-per-second (PPS) generator: A precision 1 PPS signal is generated by the TFR subsystem (see section 4.6) in the KAPB and transferred to each individual digitizer via a dedicated optical fibre. The PPS generator converts the optical signal into an electrical signal that is passed on to the ADC via a digital fibre link to allow precise time-stamping of voltage sample data. The PPS generator has an optical retro-reflector that allows precision round-trip time delay calibration of the 1 PPS signal path between the TFR subsystem at the KAPB (traceable to UTC) and the digitizers on each receptor (with a well-defined and stable offset relative to the delay centre of the receptor).

- Noise diode control: This unit provides a programmable switching signal to the receiver that is synchronized with the 1 PPS and sample clock signals.

The UHF and L-band digitizers on a given receptor connect to the receptor data switch located in the pedestal shielded compartment via four 10GbE multi-mode links (see Figure 2). Currently a single 40GbE single-mode fibre link transfers this data from the receptor switch to the CBF core switch at the KAPB (see section 4.5). The maximum fibre run from a receptor to the KAPB is $\sim 10 \mathrm{~km}$. 


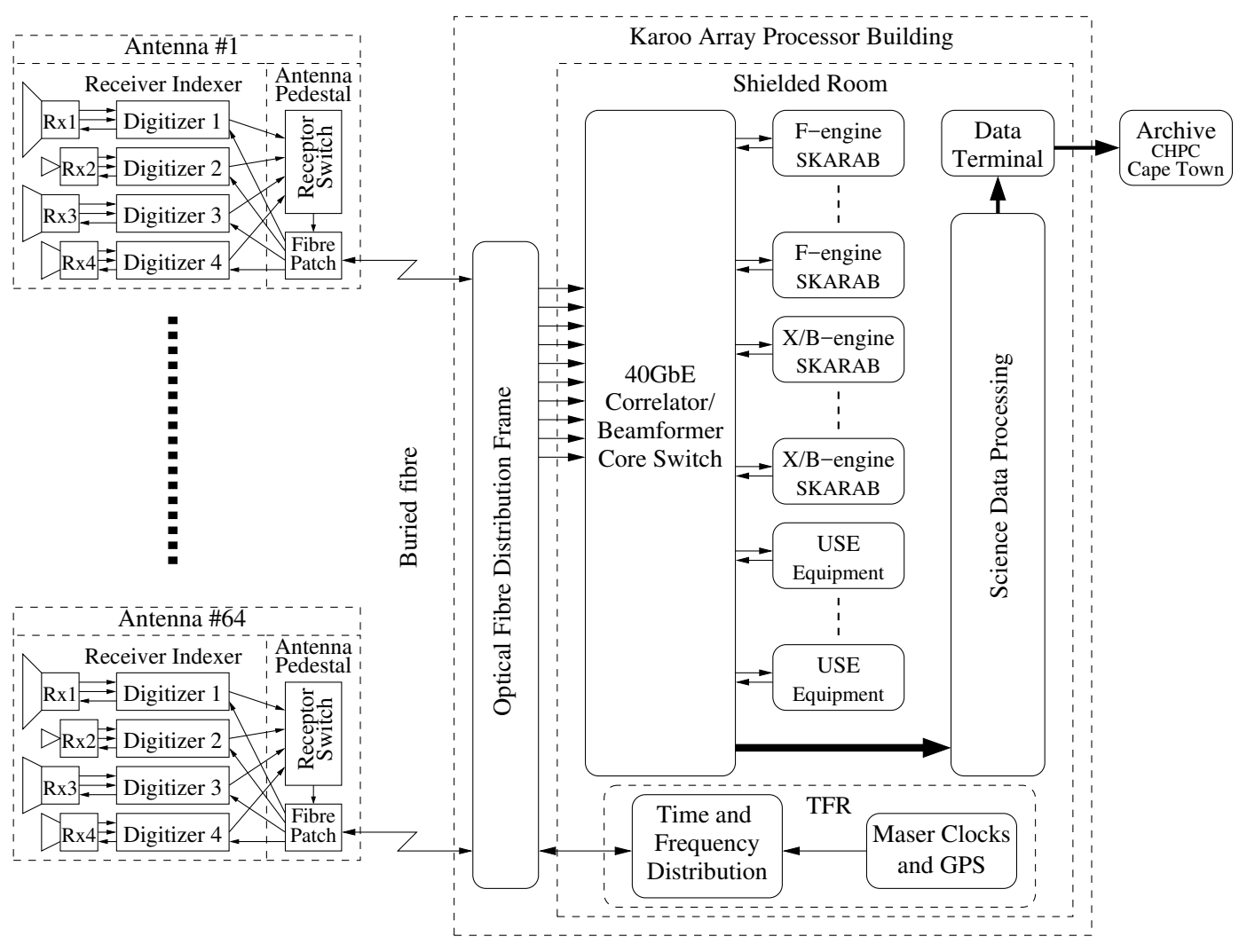

Figure 2: A block diagram showing the overall MeerKAT signal transport and data processing architecture, including analogue and digital signal paths, the digitizers, the correlator/beamformer (CBF), the science data processing (SDP) subsystem, and the time and frequency reference (TFR) subsystem. Signal transport from the receptor switches in each of the pedestals to the CBF core switch is via buried single-mode fibre-optic cables carrying $40 \mathrm{~Gb} / \mathrm{s}$ Ethernet. The SKARAB processing nodes providing the filter-bank, correlation and beamforming functions (F-engines and $\mathrm{X} / \mathrm{B}$-engines) receive and output data via bi-directional ports on the CBF core switch. Ports are available on the CBF switch that allow user-provided equipment (USE) to subscribe to various data products, and the science data processing subsystem (see Figure 3) extracts voltage and visibility data from the CBF switch. The SPEAD protocol (Streaming Protocol for Exchanging Astronomical Data) is used for the transfer high-speed data over the Ethernet links. Although not shown in this diagram, the Control and Monitoring (CAM) subsystem and associated signal paths are embedded into all of the components shown.

\subsection{Correlator/Beamformer}

The MeerKAT correlator/beamformer (CBF) subsystem implements an FX/B style central real-time signal processor. The antenna voltage data streams for both polarizations from all receptors are split into the required number of frequency channels prior to correlation (X) and/or beamforming (B) by processing nodes called "F-engines" ( $\mathrm{F}$ as in Fourier). The channelization is done by a digital polyphase filter-bank (PFB) that is optimized to provide a specified uniform frequency response across individual channels (a requirement driven by spectral line observations), while minimizing inter-channel spectral leakage (a requirement driven by pulsar timing observations). The correlation and beamforming operations are performed by processing nodes called 
"X-engines" and "B-engines" respectively (the X and B operations actually co-exist on composite $\mathrm{X} / \mathrm{B}$ engines). The channelized data is re-ordered and routed by corner-turner logic and the core switch so that individual nodes can process dual-polarization data from all receptors within a given frequency sub-band. Full Stokes processing is performed by both the X-and B-engines.

The CBF subsystem is based on the CASPER concept and architecture [6] that uses commodity network devices (the core switch) to handle digital data transfer and re-ordering between processing nodes. The core switch is implemented using a large number of commodity $40 \mathrm{GbE}$ switches arranged as a Clos crossbar. This arrangement allows for an extendable switch fabric that provides full-crossbar, non-blocking functionality, and that is multicast capable. A customized load-balancing algorithm is employed to minimize packet collisions. The processing nodes for the full MeerKAT CBF will be SKARAB boards fitted with the Virtex 7 SX690T field-programmable gate array (FPGA). The SKARAB boards can support up to $1640 \mathrm{GbE}$ ports, but for the MeerKAT CBF implementation only one $40 \mathrm{GbE}$ port per card is needed to connect to the core switch. Early array releases of MeerKAT will have a reduced capability CBF based on the ROACH 2 board that is fitted with the Virtex 6 SX475T FPGA.

The design philosophy for the CBF is to provide a moderate number of modes with limited parameterization (e.g. integration period) that cover the required functionality, rather than a large number of very specific modes, or a small number of highly-configurable modes. This strategy was based on prior experience from other telescopes and the need to maximize reliability and minimize development and commissioning effort. The MeerKAT CBF will evolve to include the following modes and data products (some of these are TBC and depend on the evolving requirements of the user community and the technical capabilities of the SKARAB platform):

- 4 096-channel, 4 Stokes visibilities, full digitized bandwidth

- 32 768-channel, 4 Stokes visibilities, full digitized bandwidth

- Four 4 096-channel tied-array beams

- Incoherent sum of antenna data

- Fly's-eye mode with 64 independent spectrometers, one per antenna

- 4 096-channel, 4 Stokes visibilities, for five independently tuneable $11.6 \mathrm{MHz}$ or $6.6875 \mathrm{MHz}$ sub-bands (TBC)

- 2 second voltage buffer data for transient capture (TBC)

- Tied array beam for VLBI

Early array releases will implement a subset of this functionality (see:

http://public.ska.ac.za/meerkat/meerkat-schedule

for the most up-to date deployment schedule).

The core switch will have spare $40 \mathrm{GbE}$ ports for the incorporation of user-provided digital back-ends that can subscribe to any of the data products flowing through the switch, including raw telescope voltages, visibilities and tied-array voltages. The Clos architecture of the core switch allows for the expansion of this facility in the future, should more user-provided equipment materialize. 


\subsection{Time and Frequency Reference}

The MeerKAT time and frequency reference (TFR) subsystem provides the time and frequency references that are needed by the various components of the telescope. The services provided by TFR include:

- Karoo Telescope Time (KTT): The KTT facility provides the 1 PPS signal that is used by the digitizers to time-stamp the voltage samples. This is a precise time service, primarily required for long-term pulsar timing experiments, that is traceable to within 5 ns of UTC. Key hardware components used to provide KTT are two hydrogen maser clocks and two timetransfer GPS receivers. The GPS common-view methodology is used to monitor, in real time, the KTT maser clocks against the UTC(ZA) clocks located at the National Metrology Institute of South Africa (NMISA). This real-time time transfer is subject to the time offset between UTC(ZA) and UTC, and high precision timing requires retrospective calibration that makes use of the monthly tables from BIPM that provide the UTC-UTC(ZA) offset. The TFR has instrumentation for measuring the round-trip delay of the 1 PPS signal to individual digitizers. The digitizer time stamp can be traced to UTC to a precision of $5 \mathrm{~ns}$ after retrospective off-line calibration correcting for the UTC(ZA)-UTC offset and the receptor/TFR 1 PPS delays.

- ADC sample clocks: The sample clocks are derived from the $5 \mathrm{GHz}$ reference frequency provided by the hydrogen maser clocks. A tree network of RF splitters, lasers and optical splitters provides the 64 phase-matched replicas of the 1 PPS and sample clock signals that are sent to the digitizers on the receptors (one set per receiver band).

- PTP Grandmaster: Two GPS-disciplined rubidium clocks provide the PTP reference that is used by telescope components such as the antenna control units and telescope control and monitoring software.

All of the critical TFR components are located in a purpose-built compartment within the KAPB shielded room that provides precision temperature control. This isolates the clocks and frequency reference hardware from the temperature cycling of the main digital equipment rack area that is a characteristic of standard data centre HVAC equipment. The hydrogen masers are mounted on pneumatic vibration damping tables to reduce acoustic modulation of the reference frequency. EMC shielding of the sample clock generation and distribution components is necessary to avoid phase noise caused by coupling to the hostile EMI environment inside the KAPB shielded room. The TFR design employs redundancy of all critical components and functions, including power supply and temperature control instrumentation, to ensure a stable and reliable KTT service.

\subsection{Control and Monitoring}

The control and monitoring (CAM) subsystem supervises the coordinated operation of all of the telescope components. The key functions of CAM include:

- Observation planning and scheduling.

- Telescope configuration and observation execution. 
- Persistence of configuration and calibration data.

- Monitoring the status of all telescope components and infrastructure elements.

- Interfaces to operators, maintainers, developers and observers.

The KATCP communications protocol is implemented on all MeerKAT subsystems so that there is a common and consistent interface with CAM. The functionality of CAM, including the available observing modes, will evolve throughout the deployment of the MeerKAT array releases.

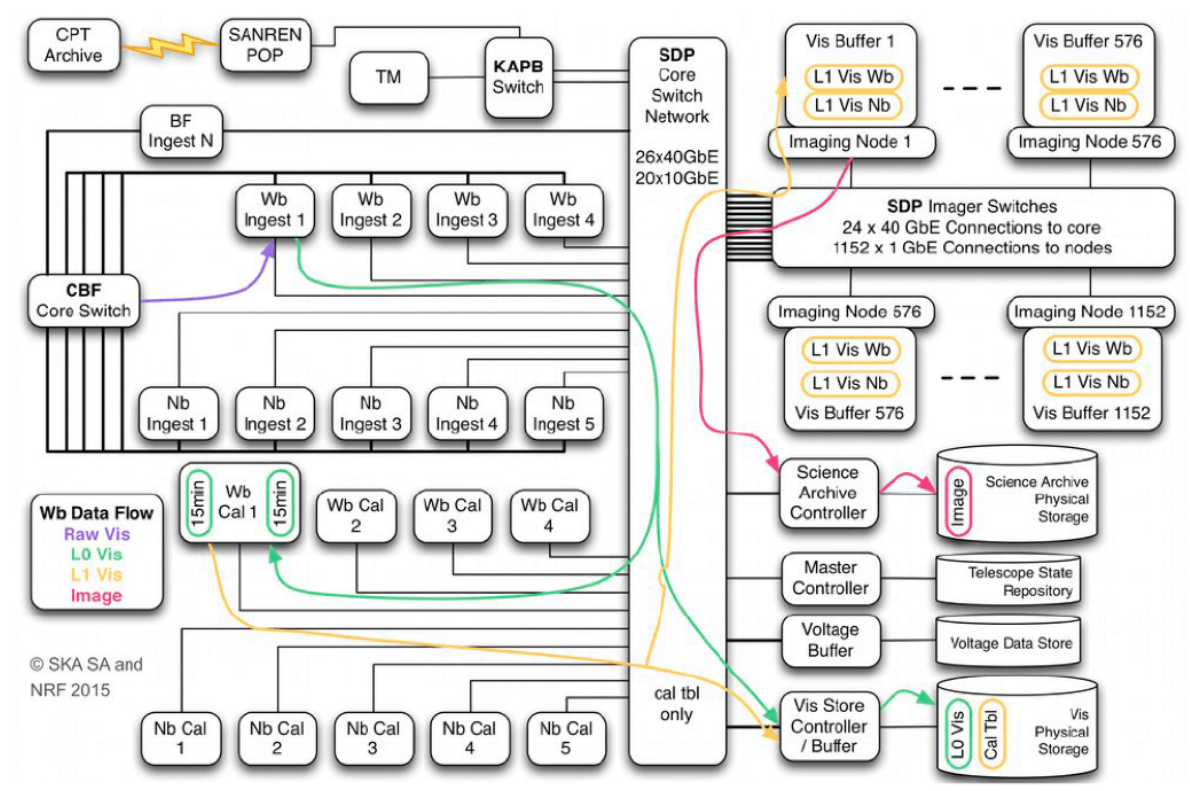

Figure 3: A block diagram of the science data processor (SDP) elements and data flow. The SPEAD protocol (Streaming Protocol for Exchanging Astronomical Data) is used for data transfer from CBF to SDP, and to export SDP data products. SDP data is stored in HDF5 format.

\subsection{Science Data Processing}

The functions of the science data processing (SDP) element include:

- Ingest and pre-processing of visibilities from CBF, including baseline dependent visibility averaging, RFI flagging, van Vleck correction and data quality monitoring.

- Voltage data capture.

- Signal displays.

- Data archiving.

- Calibration and imaging pipeline. 
Figure 3 shows the architecture of the MeerKAT SP subsystem. The processing nodes are a mixture of CPUs and GPUs, and novel processor options are currently being investigated to lower the capital and operations cost of the SDP subsystem. The SPEAD protocol (Streaming Protocol for Exchanging Astronomical Data) is used for data transfer throughout the SDP subsystem, and SDP data is stored in HDF5 format on disks and tape.

Dedicated ingest processors are assigned to the three variants of CBF data products: full digitized bandwidth visibilities ( $\mathrm{Wb}$ ), five sub-band visibilities $(\mathrm{Nb})$ and tied array (BF). This is necessary because CBF can produce all of these products simultaneously. The SP core switch directs the data from the various ingest processors to an appropriate storage location and/or calibration node. Visibilities are initially buffered in disk storage and then transferred to a tape library. The voltage data store is used for non-imaging observations and can be exported in VDIF format for VLBI experiments.

The calibration nodes use double-buffering to allow sufficient processing time to obtain robust calibration solutions in real-time. The post-calibration data is then streamed to the imaging pipeline. The imaging pipeline capitalizes on the inherently parallel nature of the data and imaging algorithms, and consists of visibility buffers, imaging nodes and a dedicated switch fabric. The final images are transferred to the science archive.

Although there will be a standard MeerKAT processing pipeline, a set of tools is being developed to allow an external user to run their own applications on the SDP hardware. These tools are based on the Docker platform.

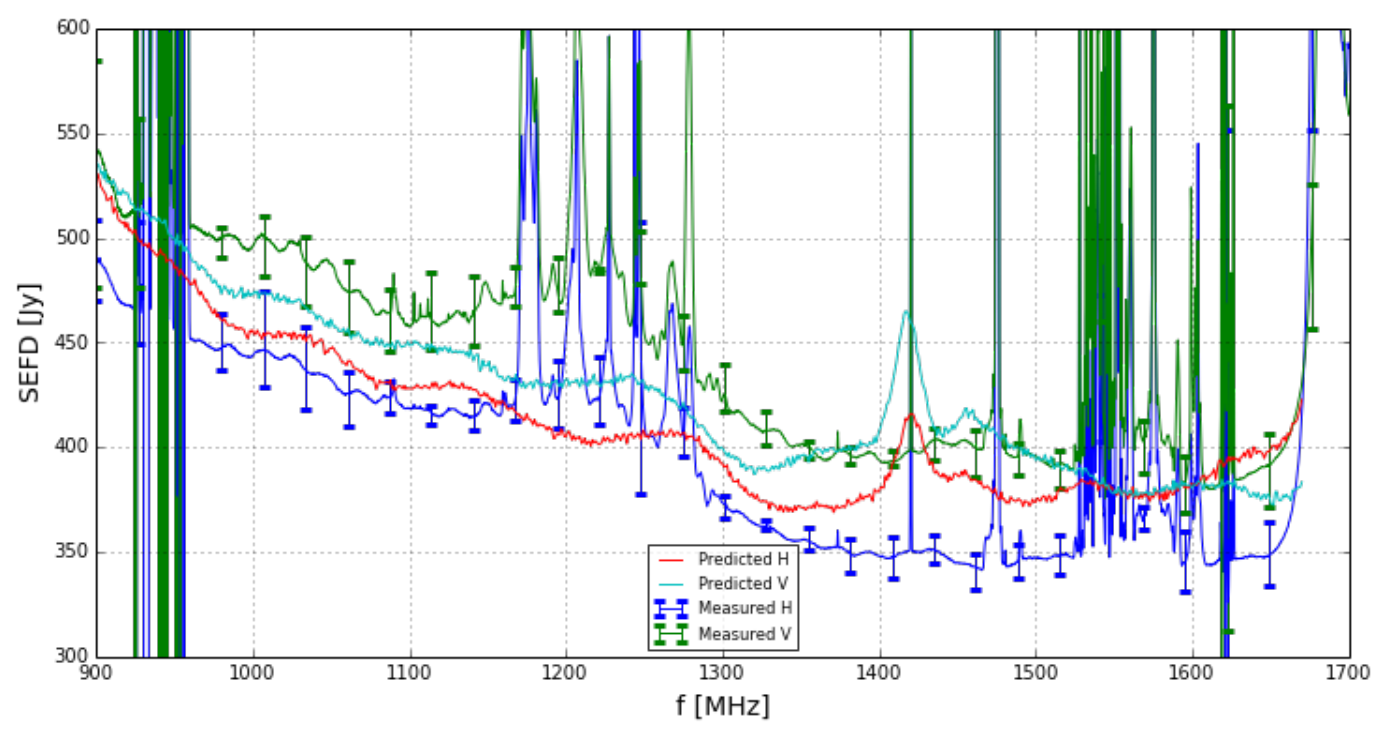

Figure 4: The spectrum of the SEFD of a single receptor at $37^{\circ}$ elevation derived from L-band measurements made of Tau A, smoothed over $1 \mathrm{MHz}$ frequency bins. The measurements were made on antenna M062 using three different L-band receivers. The error bars on the measured curves represent the total range of all measurements made with all three receivers. 


\section{Telescope Performance}

A hierarchy of tests against the required specifications and functionality are performed as individual telescope components are assembled into increasingly complex functional units. Initial acceptance tests are performed on low-level devices, and more sophisticated and extensive tests are performed as subsystem and system assembly progresses. Qualification tests are performed on subsystems to prove that the design and manufacturing processes have resulted in an article that achieves the required performance and functionality, and that this compliance is repeatable as more articles are produced. The majority of the most important MeerKAT performance specifications are allocated to the receptor, comprising the dish structure, feed horn, receiver and digitizer. For this reason this section will focus on MeerKAT receptor qualification tests.

MeerKAT was optimized for point-source sensitivity because this was a common requirement for all of the LSPs. The original specification for the MeerKAT aggregate system-equivalent flux density (SEFD) was 12.55 Jy. For the 64-element MeerKAT array this corresponds to a per-receptor SEFD of 800 Jy. Figure 4 shows the results of L-band SEFD measurements made with MeerKAT receptor M062, the second MeerKAT receptor to be installed and integrated (the first was M063). This plot shows that the SEFD is far better than the original specification across the entire L-band frequency range, and that the measured curves follow the predicted performance quite well (the predicted curves were derived from simulations and measured data for components such as LNAs). The large deviations in the measured curves are due to RFI from satellites and mobile phone base stations, and Galactic HI (at $1420 \mathrm{MHz}$ ). The measurements shown in Figure 4 were made at a relatively low elevation of $37^{\circ}$. Tipping curve measurements show that the SEFD at $15^{\circ}$ elevation is less than $20 \%$ higher than at zenith over all L-band frequencies.

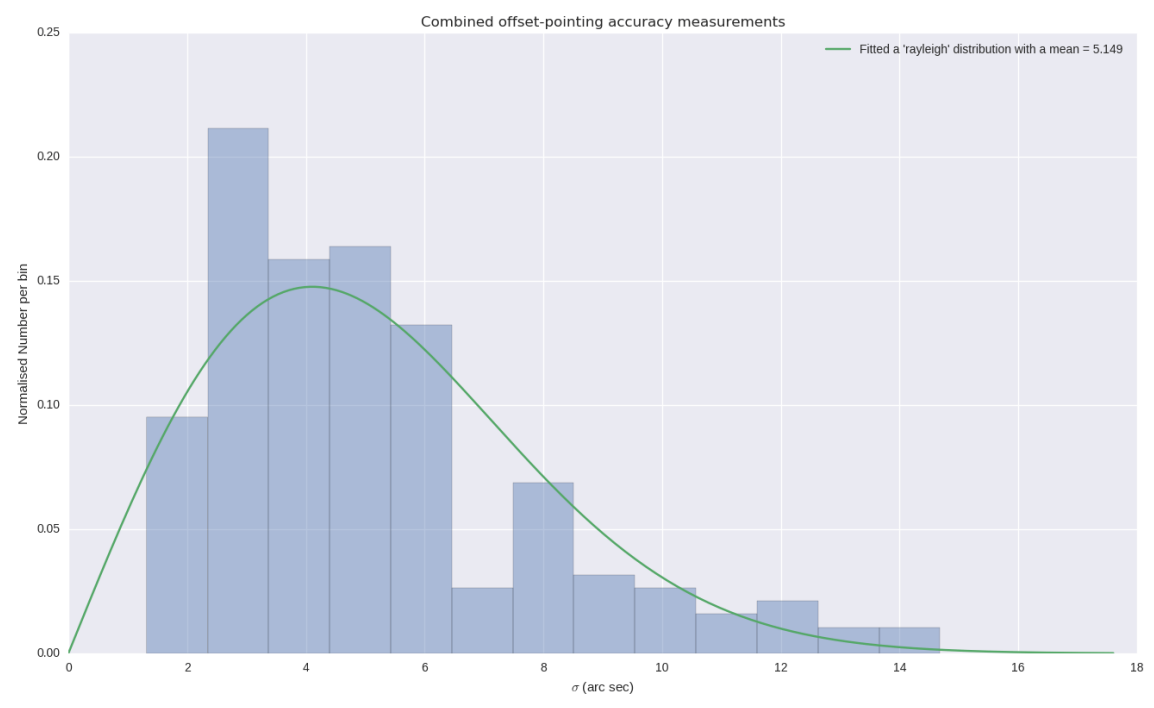

Figure 5: A histogram of all referenced pointing measurements made to date on all integrated receptors under all operating conditions.

This improvement in sensitivity over the original specification was a result of the design 
choices made at the CoDR and PDR stages of the project, specifically the choice of Gregorian offset optics, sub-octave receivers and G-M cryo-coolers. Ku-band holography measurements were made using a satellite beacon signal in order to characterize Ruze effects at higher frequeincies. These qualification tests showed that the APE exceeds $95 \%$ at $14.5 \mathrm{GHz}$, and hence exceeds the original specification. MeerKAT receptors will function well at frequencies up to and above $25 \mathrm{GHz}$.

The key receptor performance factors affecting imaging dynamic range are telescope pointing accuracy and stability, and the primary beam pattern characteristics. Figure 5 summarizes all pointing error measurements made to date for all commissioned receptors under all observing conditions (these measurements employed offset reference pointing, as defined in the receptor pointing specification). The MeerKAT receptors clearly exceed the required specification, with the mean pointing error of $\sim 5^{\prime \prime}$ equaling the specification for ideal operating conditions. Short time-scale pointing "jitter" was quantified using an optical camera to observe bright stars while tracking. Over a 10 second period the jitter was shown to be less than 2".
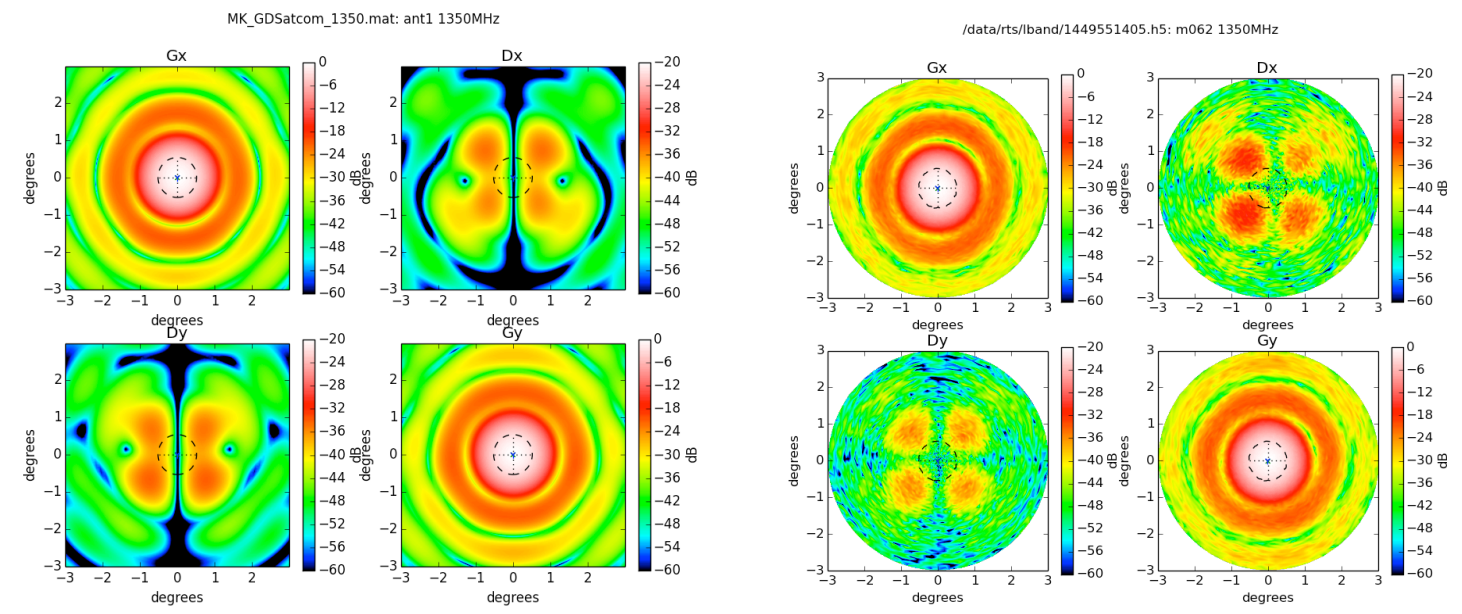

Figure 6: Comparison of the simulated MeerKAT L-band (1350 MHz) primary beam derived using electromagnetic simulations (left) and the beam of receptor M062 measured using holography. The measurement was made prior to final collimation of the reflector surfaces and feed receiver indexer.

The characteristics of the receptor L-band primary beam pattern were derived using various techniques, including holography. Figure 6 compares the beam pattern at $1350 \mathrm{MHz}$ predicted using a full-wave electromagnetic simulation against the beam pattern of MeerKAT receptor M062 measured using L-band holography (the reference source was a satellite beacon). These plots show that the measured beam pattern matches the simulations very closely, despite the fact that M062 was known to have a collimation problem at the time of the measurement. The measured patterns indicate that the second sidelobe is comfortably less than the $-23 \mathrm{~dB}$ specification, and that the cross-polarization specifications are exceeded by a large margin (in fact they exceed the goal specification). Raster scan measurements of the far sidelobes using a satellite beacon at $1549 \mathrm{MHz}$ showed that the $0 \mathrm{dBi}$ specification was exceeded over $99 \%$ of the sky, with levels of less than $-10 \mathrm{dBi}$ being recorded over a large fraction of the sky. These excellent beam characteristics vindicate the selection of the Gregorian offset optical configuration and sub-octave feeds. 


\begin{tabular}{|l|l|l|}
\hline Quantity & 2009 value & As-built value \\
\hline Number of antennas & $80(+7$ along a "spur") & 64 \\
Antenna diameter & $12 \mathrm{~m}$ & $13.965 \mathrm{~m}$ \\
Aperture efficiency & 0.7 & $0.71-0.79$ (UHF-band) \\
& & $0.75-0.81$ (L-band) \\
Field of view at $0.58 \mathrm{GHz}$ & $6 \mathrm{deg}^{2}$ & $5 \mathrm{deg}^{2}$ \\
Field of view at $1.4 \mathrm{GHz}$ & $1 \mathrm{deg}^{2}$ & $0.85 \mathrm{deg}^{2}$ \\
System temperature & $30 \mathrm{~K}$ & $27-20 \mathrm{~K}(\mathrm{UHF}-$ band) \\
& & $22-18 \mathrm{~K}(\mathrm{~L}-\mathrm{Band})$ \\
$A_{e} / T_{\text {sys }}$ (array) & $200 \mathrm{~m}^{2} / \mathrm{K}$ & $241-366 \mathrm{~m}^{2} / \mathrm{K}$ (UHF-band) \\
& & $305-339 \mathrm{~m}^{2} / \mathrm{K}$ (L-Band) \\
Minimum baseline & $20 \mathrm{~m}$ & $29 \mathrm{~m}$ \\
Maximum baseline & $8 \mathrm{~km}(60 \mathrm{~km}$ with "spur") & $7.7 \mathrm{~km}$ \\
Frequency range & $0.58-2.5 \mathrm{GHz}$ & $0.58-1.015 \mathrm{GHz}$ (UHF-band) \\
& & $0.9-1.67 \mathrm{GHz}$ (L-Band) \\
Processed band & - & $0.544-1.088 \mathrm{GHz}$ (UHF-band) \\
& & $0.856-1.712 \mathrm{GHz}$ (L-band) \\
Number of channels & 16384 & 32768 (wideband mode) \\
& & narrow-band zoom modes will \\
& & provide $\approx 0.1 \mathrm{~km} / \mathrm{s}$ resolution \\
\hline
\end{tabular}

Table 2: Key MeerKAT system parameters for UHF and L-band operation for both the 2009 reference design and the as-built telescope. The S-band receiver being provided by the Max Planck Institute for Radio Astronomy will extend the frequency coverage to $\approx 1.75-3.5 \mathrm{GHz}$.

\section{Conclusion}

The conceptualization, design, construction and commissioning of the MeerKAT telescope and associated infrastructure were based on the principles of system engineering. Key science requirements were extracted from a prioritized science case, and these requirements were converted into specifications allocated to the various telescope components. Design and technology choices were driven by rational optimization processes and critical assessment of technology maturity. Element testing and system commissioning have been thorough and rigorous. Table 2 compares the key parameters of the as-built MeerKAT against the the reference design figures presented in the original call for large survey proposals [1]. The rational methodology used during the MeerKAT design and deployment has lead to the significant increase in the telescope point-source sensitivity over the original 2009 reference value. Other changes in the telescope parameters were the result of budget constraints (e.g. maximum baseline) and technology developments (e.g. correlator channels). Construction and commissioning of MeerKAT is following the planned schedule very closely, and early user-proposed science will commence in 2018. 


\section{References}

[1] R. S. Booth, W. J. G. de Block, J. L. Jonas and B. Fanaroff, MeerKAT Key Project Science, Specifications, and Proposals (2009) [arXiv0910.2935B].

[2] J. L. Jonas, MeerKAT - The South African Array With Composite Dishes and Wide-Band Single Pixel Feeds, IEEE Proceedings 97 (2009) 1522-1530.

[3] Y. Mizugutch, M. Akagawa and H. Yokoi, Offset dual reflector antennas (Oct 1976) IEEE AP-S International Symposium Digest.

[4] R. Lehmensiek and I. P. Theron, The Design of the MeerKAT UHF Band Feed (2014) EuCAP.

[5] R. Lehmensiek and I. P. Theron, The design of the MeerKAT L-band feed (2012) ICEAA, Cape Town.

[6] J. Hickish, Z. Abdurashidova, Z. Ali, K. D. Buch, S. C. Chaudhari, H. Chen, M. Dexter, R. S. Domagalski, J. Ford, G. Foster, D. George, J. Greenberg, L. Greenhill, A. Isaacson, H. Jiang, G. Jones, F. Kapp, H. Kriel, R. Lacasse, A. Lutomirski, D. MacMahon, J. Manley, A. Martens, R. McCullough, M. V. Muley, W. New, A. Parsons, D. C. Price, R. A. Primiani, J. Ray, A. Siemion, V Van Tonder, L. Vertatschitsch, M. Wagner, J. Weintroub and D. Werthimer, A Decade of Developing Radio-Astronomy Instrumentation using CASPER Open-Source Technology (2016)

[arXiv: 1611.01826] 